\title{
Precision Muon Reconstruction in Double Chooz
}

Y. Abe ${ }^{a a}$, J.C. dos Anjos ${ }^{\mathrm{e}}$, J.C. Barriere ${ }^{\mathrm{n}}$, E. Baussan ${ }^{\mathrm{v}}$ I. Bekman ${ }^{\mathrm{a}}$, M. Bergevin ${ }^{\mathrm{i}}$, T.J.C. Bezerra ${ }^{\mathrm{y}}$, L. Bezrukov ${ }^{\mathrm{m}}$, E. Blucher ${ }^{\mathrm{f}}$, C. Buck ${ }^{\mathrm{s}}$, J. Busenitz ${ }^{\mathrm{b}}$, A. Cabrera ${ }^{\mathrm{d}}$, E. Caden ${ }^{\mathrm{j}}$, L. Camilleri ${ }^{\mathrm{h}}$, R. Carr ${ }^{\mathrm{h}}$, M. Cerrada ${ }^{\mathrm{g}}$, P.-J. Chang ${ }^{\mathrm{o}}$, E. Chauveau ${ }^{\mathrm{y}}$, P. Chimentia ${ }^{\mathrm{ae}}$, A.P. Collin ${ }^{\mathrm{s}}$, E. Conover ${ }^{\mathrm{f}}$, J.M. Conrad ${ }^{\mathrm{r}}$, J.I. Crespo-Anadón ${ }^{\mathrm{g}}$, K. Crum ${ }^{\mathrm{f}}$, A. Cucoanes ${ }^{\mathrm{w}}$, E. Damon ${ }^{\mathrm{j}}$, J.V. Dawson ${ }^{\mathrm{d}}$, J. Dhooghe ${ }^{\mathrm{i}}$, D. Dietrich ${ }^{\mathrm{ad}}$, Z. Djurcic ${ }^{\mathrm{c}}$, M. Dracos ${ }^{\mathrm{v}}$, M. Elnimr ${ }^{\mathrm{b}}$, A. Etenko ${ }^{\mathrm{q}}$, M. Fallot ${ }^{\mathrm{w}}$, F. von Feilitzsch ${ }^{\text {ac }}$, J. Felde ${ }^{\mathrm{i}, 1}$, S.M. Fernandes ${ }^{\mathrm{b}}$, V. Fischer ${ }^{\mathrm{n}}$, D. Franco ${ }^{\mathrm{d}}$, M. Franke ${ }^{\mathrm{ac}}$, H. Furuta ${ }^{\mathrm{y}}$, I. Gil-Botella ${ }^{\mathrm{g}}$, L. Giot ${ }^{w}$, M. Göger-Neffac ${ }^{\text {, L.F.G. Gonzalez }}{ }^{\text {af }}$, L. Goodenough ${ }^{c}$, M.C. Goodman ${ }^{c}$, C. Grant ${ }^{i}$, N. Haag $^{\text {ac }}$, T. Hara $^{\text {p }}$, J. Haser ${ }^{\mathrm{s}}$, M. Hofmann ${ }^{\text {ac }}$, G.A. Horton-Smith ${ }^{\mathrm{o}}$, A. Hourlier ${ }^{\mathrm{d}}$, M. Ishitsuka ${ }^{\text {aa }}$, J. Jochum ${ }^{\text {ad }}$, C. Jollet ${ }^{\mathrm{v}}$, F. Kaether ${ }^{\mathrm{s}}$, L.N. Kalousis ${ }^{\text {ag }}$, Y. Kamyshkov ${ }^{\mathrm{x}}$, D.M. Kaplan ${ }^{1}$, T. Kawasaki ${ }^{\mathrm{t}}$, E. Kemp ${ }^{\text {af }}$, H. de Kerret ${ }^{\mathrm{d}}$, D. Kryn ${ }^{\mathrm{d}}$, M. Kuze ${ }^{\text {aa }}$, T. Lachenmaier ${ }^{\text {ad }}$, C.E. Lane ${ }^{\mathrm{j}}$, T. Lasserre ${ }^{\mathrm{n}, \mathrm{d}}$, A. Letourneau ${ }^{\mathrm{n}}$, D. Lhuillier ${ }^{\mathrm{n}}$, H.P. Lima Jr ${ }^{\mathrm{e}}$, M. Lindner ${ }^{\mathrm{s}}$, J.M. López-Castañog ${ }^{\mathrm{g}}$, J.M. LoSecco ${ }^{\mathrm{u}}$, B. Lubsandorzhiev ${ }^{\mathrm{m}}$, S. Lucht ${ }^{\mathrm{a}}$, J. Maeda ${ }^{\mathrm{ab}, 2}$, C. Mariani ${ }^{\mathrm{ag}}$, J. Maricic ${ }^{\mathrm{j}, 3}$,

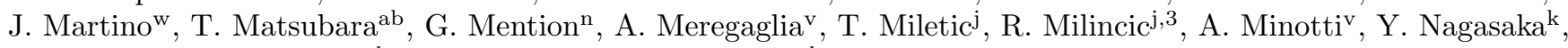
Y. Nikitenko ${ }^{\mathrm{m}}$, P. Novella ${ }^{\mathrm{d}}$, L. Oberauer ${ }^{\mathrm{ac}}$, M. Obolensky ${ }^{\mathrm{d}}$, A. Onillon ${ }^{\mathrm{w}}$, A. Osborn ${ }^{\mathrm{x}}$, C. Palomares $^{\mathrm{g}}$, I.M. Pepe $^{\mathrm{e}}$, S. Perasso ${ }^{\mathrm{d}}$, P. Pfahler ${ }^{\mathrm{ac}}$, A. Porta ${ }^{\mathrm{w}}$, G. Pronost ${ }^{\mathrm{w}}$, J. Reichenbacher ${ }^{\mathrm{b}}$, B. Reinhold ${ }^{\mathrm{s}, 3}$, M. Röhling ${ }^{\text {ad }}$, R. Roncin ${ }^{\mathrm{d}}$, S. Roth ${ }^{a}$, B. Rybolt ${ }^{x}$, Y. Sakamoto ${ }^{z}$, R. Santorellig, A.C. Schilithz ${ }^{\mathrm{e}}$, S. Schönert ${ }^{\text {ac }}$, S. Schoppmann ${ }^{\mathrm{a}}$, M.H. Shaevitz ${ }^{\mathrm{h}}$,

R. Sharankova ${ }^{\text {aa }}$, S. Shimojima ${ }^{\text {ab }}$, D. Shrestha ${ }^{o}$, V. Sibille ${ }^{n}$, V. Sinev ${ }^{\mathrm{m}}$, M. Skorokhvatov ${ }^{\mathrm{q}}$, E. Smith ${ }^{\mathrm{j}}$, J. Spitz $^{\mathrm{r}}$,

A. Stahl ${ }^{a}$, I. Stancu ${ }^{b}$, L.F.F. Stokes ${ }^{\text {ad }}$, M. Strait ${ }^{\mathrm{f}}$, A. Stüken ${ }^{\mathrm{a}}$, F. Suekane ${ }^{\mathrm{y}}$, S. Sukhotin ${ }^{\mathrm{q}}$, T. Sumiyoshi ${ }^{\text {ab }}$, Y. Sun ${ }^{\mathrm{b}, 3}$,

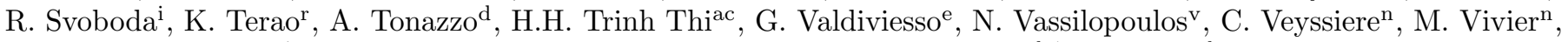

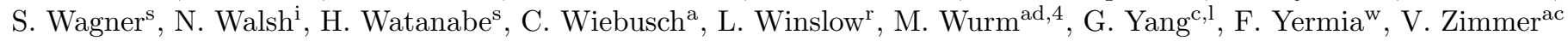

${ }^{a}$ III. Physikalisches Institut, RWTH Aachen University, 52056 Aachen, Germany

${ }^{b}$ Department of Physics and Astronomy, University of Alabama, Tuscaloosa, Alabama 35487, USA ${ }^{c}$ Argonne National Laboratory, Argonne, Illinois 60439, USA

${ }^{d}$ AstroParticule et Cosmologie, Université Paris Diderot, CNRS/IN2P3, CEA/IRFU, Observatoire de Paris, Sorbonne Paris Cité, 75205 Paris Cedex 13, France

${ }^{e}$ Centro Brasileiro de Pesquisas Físicas, Rio de Janeiro, RJ, 22290-180, Brazil

${ }^{f}$ The Enrico Fermi Institute, The University of Chicago, Chicago, Illinois 60637, USA

${ }^{g}$ Centro de Investigaciones Energéticas, Medioambientales y Tecnológicas, CIEMAT, 28040, Madrid, Spain

${ }^{h}$ Columbia University; New York, New York 10027, USA

${ }^{i}$ University of California, Davis, California 95616, USA

${ }^{j}$ Department of Physics, Drexel University, Philadelphia, Pennsylvania 19104, USA

${ }^{k}$ Hiroshima Institute of Technology, Hiroshima, 731-5193, Japan

${ }^{l}$ Department of Physics, Illinois Institute of Technology, Chicago, Illinois 60616, USA

${ }^{m}$ Institute of Nuclear Research of the Russian Academy of Sciences, Moscow 117312, Russia

${ }^{n}$ Commissariat à l'Energie Atomique et aux Energies Alternatives, Centre de Saclay, IRFU, 91191 Gif-sur-Yvette, France

${ }^{\circ}$ Department of Physics, Kansas State University, Manhattan, Kansas 66506, USA

${ }^{p}$ Department of Physics, Kobe University, Kobe, 657-8501, Japan

${ }^{q}$ NRC Kurchatov Institute, Moscow 123182, Russia

${ }^{r}$ Massachusetts Institute of Technology, Cambridge, Massachusetts 02139, USA

${ }^{s}$ Max-Planck-Institut für Kernphysik, 69117 Heidelberg, Germany

${ }^{t}$ Department of Physics, Niigata University, Niigata, 950-2181, Japan

${ }^{u}$ University of Notre Dame, Notre Dame, Indiana 46556, USA

${ }^{v}$ IPHC, Université de Strasbourg, CNRS/IN2P3, 67037 Strasbourg, France

${ }^{w}$ SUBATECH, CNRS/IN2P3, Université de Nantes, Ecole des Mines de Nantes, 44307 Nantes, France

${ }^{x}$ Department of Physics and Astronomy, University of Tennessee, Knoxville, Tennessee 37996, USA

${ }^{y}$ Research Center for Neutrino Science, Tohoku University, Sendai 980-8578, Japan

${ }^{z}$ Tohoku Gakuin University, Sendai, 981-3193, Japan

${ }^{a a}$ Department of Physics, Tokyo Institute of Technology, Tokyo, 152-8551, Japan

${ }^{a b}$ Department of Physics, Tokyo Metropolitan University, Tokyo, 192-0397, Japan

${ }^{a c}$ Physik Department, Technische Universität München, 85748 Garching, Germany

${ }^{a d}$ Kepler Center for Astro and Particle Physics, Universität Tübingen, 72076 Tübingen, Germany

ae Universidade Federal do ABC, UFABC, Santo André, SP, 09210-580, Brazil

${ }^{a f}$ Universidade Estadual de Campinas-UNICAMP, Campinas, SP, 13083-970, Brazil

${ }^{a g}$ Center for Neutrino Physics, Virginia Tech, Blacksburg, Virginia 24061, USA

\footnotetext{
${ }^{1}$ Now at Department of Physics, University of Maryland, College Park, Maryland 20742, USA.

${ }^{2}$ Now at Department of Physics, Kobe University, Kobe, 657-8501, Japan.

${ }^{3}$ Now at Department of Physics \& Astronomy, University of Hawaii at Manoa, Honolulu, Hawaii 96822, USA.

${ }^{4}$ Now at Institut für Physik and Excellence Cluster PRISMA, Johannes Gutenberg-Universität Mainz, 55128 Mainz, Germany.
} 


\begin{abstract}
We describe a muon track reconstruction algorithm for the reactor anti-neutrino experiment Double Chooz. The Double Chooz detector consists of two optically isolated volumes of liquid scintillator viewed by PMTs, and an Outer Veto above these made of crossed scintillator strips. Muons are reconstructed by their Outer Veto hit positions along with timing information from the other two detector volumes. All muons are fit under the hypothesis that they are through-going and ultrarelativistic. If the energy depositions suggest that the muon may have stopped, the reconstruction fits also for this hypothesis and chooses between the two via the relative goodness-of-fit. In the ideal case of a through-going muon intersecting the center of the detector, the resolution is $\sim 40 \mathrm{~mm}$ in each transverse dimension. High quality muon reconstruction is an important tool for reducing the impact of the cosmogenic isotope background in Double Chooz.
\end{abstract}

Keywords: Double Chooz; muon reconstruction; neutrino detector

\section{Introduction}

Double Chooz is a reactor anti-neutrino experiment designed to measure the mixing parameter $\theta_{13}$ by observing inverse beta decay events, $\bar{\nu}_{e} p \rightarrow e^{+} n$. The prompt positron and delayed capture of the neutron form the signal. The design details of the detector have been described elsewhere [1]. Here, the aspects important for muon reconstruction are given. The detector consists of four concentric cylindrical volumes and the Outer Veto (OV). The inner three volumes form a single optical volume isolated from the fourth and are collectively called the Inner Detector (ID). The four detector volumes are:

1. The Neutrino Target (NT), an innermost volume of gadolinium-loaded scintillator in an acrylic vessel $2.4 \mathrm{~m}$ in height and diameter. The gadolinium is used to decrease the time delay and increase the observed energy of neutron capture.

2. The Gamma Catcher (GC), surrounding the NT, a volume of unloaded scintillator in an acrylic vessel of height and diameter $3.5 \mathrm{~m}$. For muon reconstruction purposes, the NT and GC are treated as a single undifferentiated volume, as the acrylic separating them is only $8 \mathrm{~mm}$ thick and the light output from the two scintillators is similar.

3. Outside the GC is the Buffer, a volume of nonscintillating oil in a steel vessel $5.6 \mathrm{~m}$ in height and diameter in which 390 10-inch PMTs [2, 3, 4] are placed; this volume shields the scintillator both from external backgrounds and PMT radioactivity. The PMTs are all aligned to point at the center of the NT. Each PMT is enclosed in a mu-metal shield resulting in a viewing angle of about $140^{\circ}$. On average, the distance from the PMT photocathodes to the GC is $0.7 \mathrm{~m}$.

4. The Inner Veto (IV), a $0.5 \mathrm{~m}$ thick volume of scintillator outside the Buffer. In this volume are 78 8-inch PMTs. These PMTs are arranged to maximize muon vetoing efficiency [5], see Fig. 1.

Above these is the OV, a segmented plastic scintillator detector. It has a $13 \mathrm{~m} \times 7 \mathrm{~m}$ lower panel of modules $1.1 \mathrm{~m}$ above the IV, and a $7 \mathrm{~m} \times 3 \mathrm{~m}$ upper panel $3.9 \mathrm{~m}$ above the lower. Each OV module is made of two layers of 32 scintillator strips, either $3.2 \mathrm{~m}$ or $3.6 \mathrm{~m}$ long, $50 \mathrm{~mm}$ wide, staggered by half a strip width so as to provide position information in $25 \mathrm{~mm}$ steps. In both the upper and lower $\mathrm{OV}$, two perpendicular layers of modules are used so as to provide $x$ and $y$ coordinates. When a muon crosses both the upper and the lower panels, a high-resolution track can be reconstructed. However, since the upper OV is much smaller than the lower OV, most muons that hit the OV intersect only the lower.

Due to its overburden, 300 meters water equivalent, the Double Chooz far detector has a muon rate of $46 \mathrm{~Hz}$ through the IV and $13 \mathrm{~Hz}$ through the GC. The forthcoming near detector will have a muon rate some 5 times higher. Consequently, excellent reconstruction of muons is very helpful for suppressing cosmogenic backgrounds. The most important of these are ${ }^{9} \mathrm{Li}$ and ${ }^{8} \mathrm{He}$, which are $\beta$-n emitters. With lifetimes of $257 \mathrm{~ms}$ and $172 \mathrm{~ms}$, respectively, a simple time cut after each muon cannot be used to remove them. They are produced at an average distance of $500 \mathrm{~mm}$ from a muon [6], and therefore can be rejected with high efficiency if the reconstruction resolution for both the muon and the subsequent event are good enough.

A muon reconstruction can also be used to:

- Obtain a $\mathrm{d} E / \mathrm{d} x$ measurement for muons. This can be correlated to cosmogenic isotope production and used in addition to the track position itself.

- Study cosmogenic production by stopping muons. ${ }^{12} \mathrm{~B}$ is known to be produced by stopping muons, but this process has not yet been observed for ${ }^{9} \mathrm{Li}$ or ${ }^{8} \mathrm{He}$.

- Discriminate between single muon events and more complex events of similar total energy. Notably, we can separate a muon passing through the upper corner of the IV from an accompanying fast neutron interaction in the ID.

- Image certain aspects of the detector itself in situ. For instance, we used muons to verify our photogrammetric survey of the OV. 


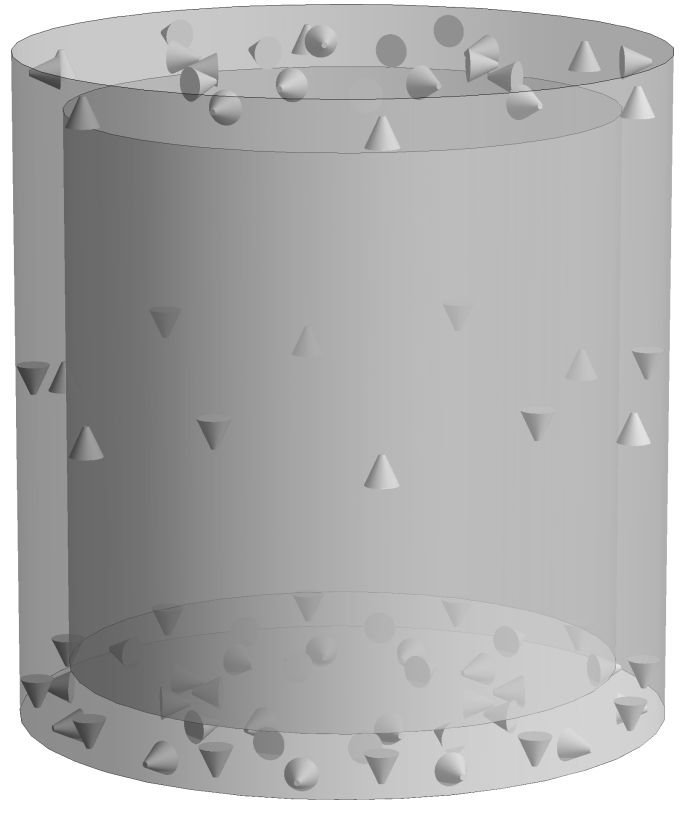

Figure 1: Layout and orientation of IV PMTs. The cones represent the PMTs to scale, with the photocathodes being at the large ends. The inner cylinder is the Buffer vessel; detail of the ID is not shown. The PMTs alternate directions for maximum vetoing efficiency.

- Perform continuous timing calibration on all PMTs.

The characteristics of a muon event depend on which detector volumes the muon intersects. Consider the case of most interest for identifying cosmogenic isotope production, muons that pass through the NT. The muon, as it traverses the ID, goes through the Buffer first and emits primarily Cherenkov light. It then crosses the GC and NT; while it does so, it emits scintillator light isotropically. Finally, it leaves the GC and traverses the Buffer again.

Muons crossing only the IV and the Buffer pass through no scintillator in the ID. The buffer oil emits Cherenkov light and most likely a small amount of scintillation light as well. Because of these two sources of light and because the scintillator in the GC absorbs the directional Cherenkov light and re-emits it isotropically, the overall pattern of light is more complex than a simple Cherenkov cone. A muon that intersects the GC but not the NT may, depending on its path length in the GC, more closely resemble either of the above two cases.

A muon typically crosses the IV twice. However, at the center of the top of the IV is the chimney through which the ID volumes were filled and calibration sources are inserted. About $0.5 \%$ of muons crossing the ID pass through enough of the chimney at the top of the IV to substantially reduce their IV signal. Additionally, about $2 \%$ of muons stop in the ID and therefore avoid the second IV crossing. A muon can also cross only the IV. All combinations are handled by the reconstruction, with the exception of muons that stop in the Buffer or IV.
In any of these situations, the muon may or may not cross the OV and, if it does, it can cross either or both OV layers. All combinations are handled.

Previous muon reconstruction algorithms for Double Chooz used either the ID or IV data alone [1, 7]. This reconstruction uses data from all detector components simultaneously. The reconstruction algorithm proceeds as follows:

1. The pulses of the IV and ID PMTs are reconstructed as described in Section 2.

2. OV-ignored fit: The event is fit ignoring OV data using the $\chi^{2}$ function described in Section 3. The fit strategy is given in Section 4.1.

3. OV-inclusive fit: If there are OV hits, the event is fit again with the OV as a constraint using the strategy given Section 4.2 .

4. If the event passes loose cuts for identifying a stopping muon, a fit under this hypothesis is done with and without use of OV data. This is described in Section 4.3 .

5. Among the fits performed, one result is chosen as the best using the criteria described in Section 5 .

The timing self-calibration for this reconstruction is described in Section 6. The resolutions achieved for various scenarios are given in Section 7 . Section 8 concludes.

\section{Pulse Reconstruction and Selection}

Each ID and IV PMT in Double Chooz is read out using a $500 \mathrm{MHz}$ 8-bit flash-ADC [8]. The readout window is $256 \mathrm{~ns}$. Waveforms for all ID and IV PMTs are recorded for each trigger. The ID is optimized for observation of 1$10 \mathrm{MeV}$ neutrino events rather than $\sim 1 \mathrm{GeV}$ muon events, and so the typical waveform from a muon candidate exceeds the range of the ADC and does not return to baseline by the end of the readout window. The IV PMTs are tuned similarly to obtain high vetoing efficiency. To account for these facts, a special pulse reconstruction is used for muons that is separate from that used for other events. This reconstruction defines a start time with an error, a rise time and total integrated charge for each pulse.

The start time of PMTs is defined as when the wave reaches halfway between the baseline and its maximum value. If the pulse saturates the $\mathrm{ADC}$, the maximum value is simply taken to be the saturation point. The time at which the pulse crosses the halfway point is interpolated based on the ADC values of the two time bins that bracket the point. This time is then corrected using the per-PMT muon-calibrated time offsets (see Section 6).

The rise time is defined as the time taken for the pulse to go from $10 \%$ to $90 \%$ of the way from the baseline to the maximum, with the same definition of maximum as with the start time. The $10 \%$ and $90 \%$ times are also interpolated as above. 
The total integrated charge for a pulse is defined as the baseline-subtracted sum of the samples, after corrections to account for the parts of the pulse lost due to the ADC range and the width of the readout window. In the case of very large pulses in which the $\mathrm{ADC}$ is still saturated at the end of the window, the total saturation time is estimated using that of neighboring PMTs and the charge is assigned accordingly.

From the rise time and charge, an error (typically between 0.6 and $2.0 \mathrm{~ns}$ ) is assigned to the start time of each tube. This error is drawn from a hardcoded table which was created through an investigation of muon fit residuals for scintillation-dominated events. A correction, as a function of rise time and charge, is then applied to the start time. The pulses with the smallest timing errors are those with fast rise times and moderate charges. These are interpreted as hits free or nearly free of Cherenkov light. Cherenkov light usually precedes the scintillator light and so lengthens the rise time while making the start time less clear. Hits with fast rise times are relatively near the muon so that they rapidly accumulate many photons produced during the first $\sim 0.5 \mathrm{~ns}$ of scintillation. PMTs with unusually large charges nearly always have rapid rise times, but since their waveform is severely clipped by the ADC it is difficult to accurately reconstruct their start time and so have errors around $1.2 \mathrm{~ns}$.

Before the fit is run, a subset of the hits are selected for use. The criteria for selecting these hits are different for the ID and IV. In the IV, the muon may or may not be directly visible by any given PMT. Most PMTs will see light, but the majority of this light is reflected. To select PMTs that see direct light, only those with pulses that saturate the ADC and with a rise time of less than $8 \mathrm{~ns}$ are accepted. The saturation requirement selects PMTs near the muon while the rise time requirement excludes pulses formed from several reflections arriving with enough total light to meet the saturation requirement.

In the ID, if the total amount of light corresponds to at least $75 \mathrm{MeV}$ deposited in scintillator, only pulses that saturate the ADC are used. This sample of events corresponds to muons which almost certainly intersect the ID scintillator, as opposed to Buffer Cherenkov-only muons. Furthermore, a cut on lower energy pulses, increasing as a function of total energy in the ID, is applied. This excludes PMTs far from the muon which tend to worsen the fit. Overall, this selection strategy removes the majority of hits due primarily to Cherenkov light which would distort the fit for events where the majority of light is from scintillation.

When the total amount of light corresponds to less than $75 \mathrm{MeV}$ deposited in ID scintillator, whether the muon produced more Cherenkov light or scintillation light depends on its trajectory. In this case, all saturated pulses are accepted. In addition, smaller pulses are accepted if they pass a cut which is a function of total event energy. This cut accepts no additional pulses at $75 \mathrm{MeV}$ and pulses as small as $15 \%$ of the saturation point at the lowest total

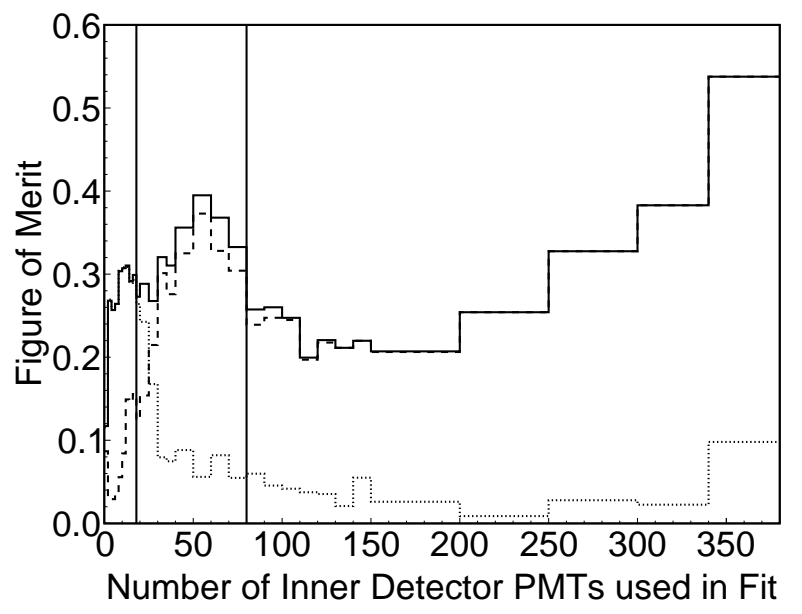

Figure 2: Methods of choosing between the Cherenkov-dominated and scintillation-dominated models. The figure of merit is the fraction of reconstructed tracks that intersect the $\mathrm{OV}$ within $0.5 \mathrm{~m}$ of the best position given by the OV. The dotted (dashed) line gives the figure of merit if all events are assumed to be Cherenkov (scintillation)dominated. The solid line gives the case both are tried and the lower $\chi^{2}$ accepted. The vertical solid lines show the cut-offs described in the text.

energies. Using data, this has been tuned for best performance as a function of energy across the $0-75 \mathrm{MeV}$ range. At the lower total-energy end this maximizes the statistics available for the fit while producing a clean sample of PMTs hit by direct Cherenkov light, excluding reflections from the vessel walls, re-emission from the wavelength shifters in the GC, and possible scintillation light in the Buffer. While we do not have a measurement of how much scintillation light the Buffer oil produces, the fact that this procedure produces good fits tells us that it is subdominant to the Cherenkov light.

\section{Fit Function}

Both the OV-ignored fit and OV-inclusive fit use the same $\chi^{2}$ fit function, which MINUIT [9] minimizes. The contribution to the $\chi^{2}$ from the ID is calculated either under the assumption of light mostly from Cherenkov or mostly from scintillation, depending on the event characteristics. The $\chi^{2}$ is built from the selected PMTs' pulse start times and their associated errors, with unselected PMTs ignored. When OV hits are present, they are used only as a spatial constraint with timing information discarded. This is because the OV uses 16 ns time bins, with timing significantly complicated by the long length of the scintillator strips. In most cases, this timing would not add any significant information.

Since one of the goals of muon reconstruction is to look for muons with high $\mathrm{d} E / \mathrm{d} x$, we do not use the total reconstructed energy in the fit function for through-going muons. There are a few exceptions to this which will be described later. 


\subsection{Choice of ID Model}

The first step of the reconstruction is to decide whether to assume that the ID is dominated by scintillation light, because the muon traversed a significant length of the GC, or instead dominated by Cherenkov light. A different function is used for the ID component of the $\chi^{2}$ fit in each of these two cases. The IV is handled the same way in either case.

If the number of selected ID PMTs is fewer than 18 ( $\lesssim 30 \mathrm{MeV}$ in scintillator-equivalent light), the code assumes a Cherenkov-dominated event. If there are more than $80(\gtrsim 180 \mathrm{MeV})$, instead the event is assumed to be scintillation-dominated. Between these, it tries both hypotheses and chooses the one that produces the smaller $\chi^{2}$. As shown in Fig. 2, the $\chi^{2}$ effectively chooses the better solution. These cut-off values were chosen to cover the cross-over region without wasting time trying hypotheses unlikely to be chosen.

\subsection{ID Model for Scintillator-dominated Events}

The NT and GC form a cylinder of scintillator. Extending from the top of the GC is the chimney, two concentric clear acrylic tubes, with outer radius $188 \mathrm{~mm}$, containing GC and NT scintillator. While light from the chimney scintillator can contribute to the observed event, it is rare for a muon to pass though a significant amount of it. It is therefore ignored for purposes of muon reconstruction. The ID scintillator is modeled as a simple cylinder with the dimensions of the GC.

Given a particular muon trajectory, we calculate when each PMT's pulse start time should be and compare to the observed time to form the $\chi^{2}$. As the muon traverses the scintillator it emits light isotropically. Due to the muon's motion, this light forms a cone similar to that of Cherenkov radiation. In addition to the cone, a sphere of light is produced behind the point where the muon enters the scintillator. The same occurs at the exit point. This is shown in Fig. 3. There are, then, three regimes to consider:

(1) The PMT sees light first from the point at which the muon enters scintillator. As can be seen in Fig. 3, a large fraction of the PMT's typically fall in this category.

(2) The first light comes from some point along the muon's track through the scintillator, i.e. the scintillation cone intersects these PMTs.

(3) The PMT's first light comes from the point at which the muon exits the scintillator.

A clean separation of PMT's into these three categories is an idealization which turns out to be too far from the truth to be used without modification. It ignores Cherenkov light and assumes arbitrarily bright scintillation. Two changes are therefore made:

First, while Cherenkov light is generally ignored for this category of events, the PMTs near the muon's exit point are very close to the muon and see much more of this light

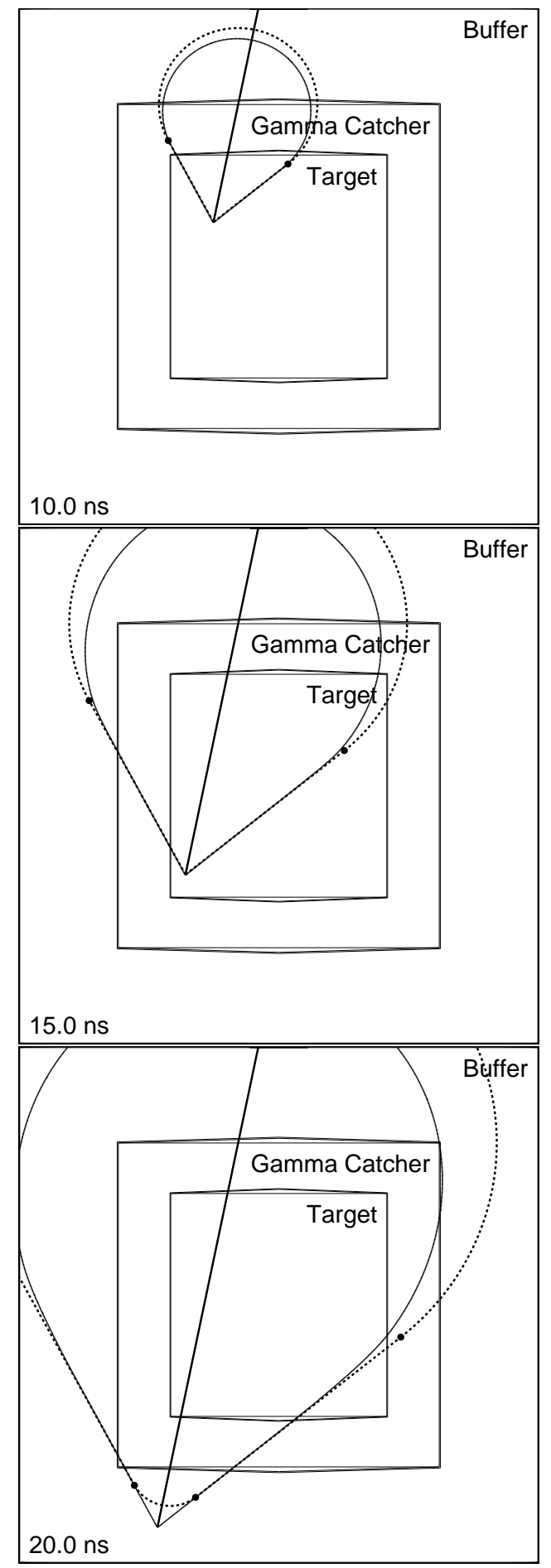

Figure 3: The wavefront of scintillator light emitted by a muon as it traverses the ID. The dashed line denotes the earliest scintillation photons. The transitions between the conical and spherical wavefronts are marked with black dots. The solid line gives the effective wavefront as used by the reconstruction. As described in the text, this lags the first scintillation photons in the region above the scintillator, and leads it below where Cherenkov light is significant. 
than others. Their observed timing is often due to Cherenkov light alone. As a reasonable approximation, we simply continue to model the cone of light after the muon has left the GC, i.e. case (3) is collapsed into case (2). Since this affects only on order of 10 PMTs in most cases, no attempt is made to adjust the cone parameters to reflect the different characteristics of Cherenkov light. A similar detector with a higher density of PMTs would benefit from an effort to resolve the Cherenkov and scintillation light separately in this region.

The second change is more involved and is necessary due to the sharp boundary between cases (1) and (2) above. This sharpness is physically unrealistic, since it implies that an infinitesimal volume of scintillator at the entry point produces enough light to be seen by every PMT that lies above the region intersected by the scintillation cone. In reality, each tube must have received light from a significant length of the muon track before it crosses the starttime threshold. To model this, we compute an effective position in the scintillator for each tube that represents where its first light was produced. As compared to the point in the scintillator that represents the earliest possible light seen by a given tube, this effective position is shifted away from the edge of the scintillator if the ideal position is at or close to the edge. The farther the ideal position is to the edge, the smaller the shift. If the ideal position is sufficiently deep in the scintillator, no shift is applied.

The functional form used to calculate these shifts is not derived from first principles, but simply designed to make a smooth transition between case (1) in which a shift is needed, and positions deep in the scintillator where we do not modify the position. It was chosen to be both reasonably fast to compute and to result in start time predictions that are generally differentiable with respect to all track parameters so that MINUIT does not see sudden changes in the $\chi^{2}$ function.

Let $l$ be the shift applied in case (1) in which the ideal first-light point is the edge of the scintillator. This is the largest shift that we impose. This length is proportional to the square of the distance between the scintillator edge and the PMT. We will apply a shift between $l$ and zero if the ideal position is between zero and $L$ from the edge of the scintillator, where $L=\pi l /(\pi-2)$. The new position is

$$
x^{\prime}=l+(L-l)\left(1-\cos \frac{\pi x}{2 L}\right),
$$

where $x$ is the distance from the edge of the scintillator to the ideal position. If the ideal position is farther from the scintillator entry point than $L$, the position is not modified.

If $L$ is greater than half of the length of the track through scintillator, $s$, the procedure is modified to prevent the effective position from being shifted past the halfway point:

$$
x^{\prime}=l+\left(\frac{s}{2}-l\right)\left(1-\cos \frac{\pi x}{s}\right),
$$

unless $l$ is also greater than half of $s$, in which case, we use simply

$$
x^{\prime}=\frac{s}{2}
$$

Because muons of different energies produce different amounts of light per unit scintillator length, there is no single correct value of the proportionality constant, the transition length parameter, that determines $l$. Higher energy muons have a higher $\mathrm{d} E / \mathrm{d} x$ and therefore a shorter transition length. It can also be substantially shorter if a muon showers near the top of the detector or the muonlike event is actually two closely spaced muons from the same air shower. Therefore, this parameter is allowed to float in the fit. In typical situations, $l \approx 300 \mathrm{~mm}$, which delays the modeled PMT start time by $\sim 1 \mathrm{~ns}$.

Once the effective position of the first light emission seen by the PMT is calculated, the expected PMT start time is calculated. Because we can achieve precision similar to the size of the PMTs, modeling them as point objects is insufficient. We instead make an approximation that keeps the computational cost low while accounting for both the size of the photocathode and the viewing angle available given the mu-metal shields. As shown in Fig. 4, the photocathodes are modeled as spheres of half the actual radius of the photocathodes, and the shields are not explicitly modeled. The centers of the model photocathodes are aligned with the centers of the real photocathodes. It is implicitly calculated where on this model of the photocathode light will strike first by calculating the time for light to reach the center of the sphere and then subtracting off the radius. This treatment has nearly the same computational cost as modeling the PMT as a point while consistently handling a variable speed of light, as discussed below. It gives substantially better resolution than approximating the PMT as a point at the center of the photocathode, or using a sphere with the full radius of the photocathode.

As with the transition length parameter, there is no single correct speed of light. The speed is a function of wavelength, and so is affected by the initial spectrum produced in each detector liquid, the reemission of this light by wavelength shifters in either the same volume or another, the wavelength-dependent attenuation, and the wavelengthdependent PMT sensitivity. Therefore, it is allowed to float in the fit, although it is constrained to stay within a reasonable range. This produces a single effective speed of light for the particular track being reconstructed. It should be noted that the effect of this speed parameter is primarily to adjust the opening angle of the scintillation cone. Because the apparent opening angle is distorted by the variation in light intensity seen by the various PMTs, even after corrections described in section 2 , this is not an effective way to measure the true speed of light.

In all, there are seven free parameters in the scintillation-dominated fit: four spatial parameters that define the track itself, the muon entry time, the transition length and the speed of light. 


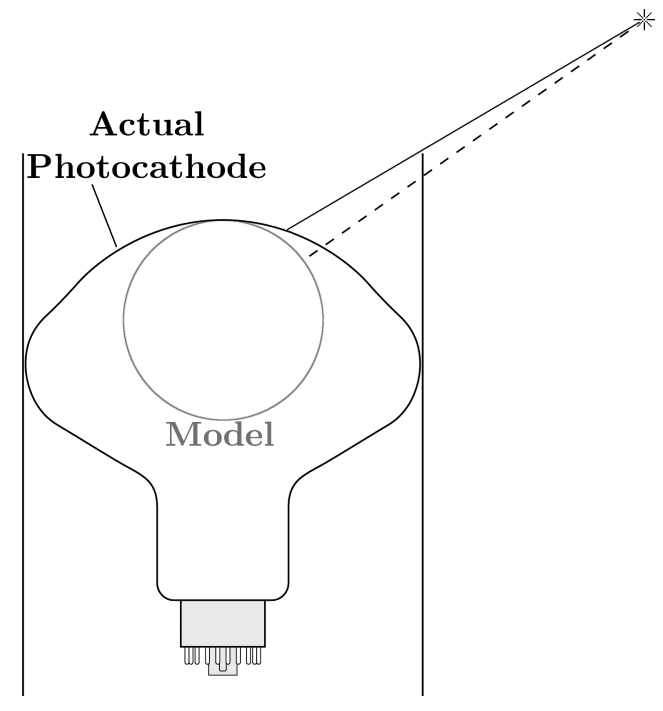

Figure 4: ID PMT with mu-metal shield. The gray circle shows the model of the photocathode used by the reconstruction to produce approximately the correct timing without the need to explicitly model the shield. For an example source of light shown in the upper right, the solid line shows the true path of light to the photocathode. The dashed line shows the modeled path, which is of nearly the same length.

\subsection{ID Treatment for Cherenkov-dominated Events}

The Cherenkov fit is substantially simpler. While the complete pattern of Cherenkov light is complicated due to absorption and re-emission in the GC, by having selected only the PMTs with the highest charge using the procedure in Section 2, usually there is only a small line or disk of PMTs on one wall of the ID that have seen direct, intense Cherenkov light. The threshold for accepting pulses has been tuned using the data to optimize the resolution by using as many pulses as possible without accepting ones due to indirect light.

The expected PMT start time for each PMT is calculated by finding the position along the track from which Cherenkov light directed at the PMT was emitted, initially ignoring the boundaries of the detector. The initial $\chi^{2}$ is based on this time. We then check, for each PMT, that the light is coming from in front of the tube. Since, unlike the scintillator case, the light is directional, if this is violated, the PMT should not see any light at all. If the light is coming from the wrong direction, a $\chi^{2}$ penalty term is applied that is zero if the light is coming in orthogonal to the PMT axis and rises as the square of the cosine of the angle as the direction becomes more backwards. In this way, the track is steered into the correct general location so that the main timing component of the $\chi^{2}$ can be usefully minimized.

To constrain the track to the correct region of the detector, the Cherenkov-dominated fit also has two additional overall penalty terms, one that prevents the track from leaving the Buffer entirely, assuming that at least one ID PMT was selected for the fit, and another that prevents it from crossing an unrealistic length of GC scintillator. Since a significant amount of scintillator can be crossed while leaving the event with primarily Cherenkovlike characteristics, this second penalty does not begin until $500 \mathrm{~mm}$ of the GC is crossed.

The Cherenkov-dominated fit does not allow the speed of light to float. Because of the small number of PMTs used, and the close proximity of these PMTs to the muon track, the fit would not sufficiently constrain the speed. It therefore has only five free parameters.

\subsection{Model}

Under either fit hypothesis, IV timing information is used in the same way. Since the IV is relatively thin, an approximation is used in which the scintillation light is treated as coming from a single point for the muon's entry position and another for its exit. These points are found by computing the intersection of the track with a cylinder that lies halfway between the Buffer vessel and the IV vessel. If the track does not intersect this cylinder because it only clips a corner or grazes the edge, a single point is used instead, centered on the intersection with the IV. For simplicity, the smaller IV PMTs are modeled as point objects with a PMT's position taken to be the center of its photocathode.

The first light observed by a PMT can be either from the muon's first or second crossing of the IV. The code considers each possibility and chooses the one that has a better $\chi^{2}$ including these contributions:

- The $\chi^{2}$ from timing, taking the expected arrival time of light to be the simple distance from the light production point to the PMT.

- A penalty term that adds to the $\chi^{2}$ if the line connecting the light production point to the PMT goes through the Buffer vessel. This penalty is a function of the length of Buffer intersected and rises smoothly from zero for zero length.

- A penalty term that checks whether the light comes from behind the PMT. Similarly, this penalty rises smoothly from zero as the angle between the PMT axis and the light direction increases from $90^{\circ}$.

Usually the fit does not explicitly constrain the track to be near the selected PMTs. This would, in general, be detrimental because the PMTs are not uniformly distributed and because the way that PMTs are chosen does not necessarily select the nearest ones. However, when the total number of PMTs in the fit falls below the number of free parameters in the fit, proximity to IV PMTs is added as a fourth penalty term. In this case an additional $\chi^{2}$ term is also used that constrains the track to have a length in the IV scintillator consistent with the total IV charge, assuming a minimum ionizing muon.

Additional penalties are applied if the track crosses the ID volume in a way severely inconsistent with the signal 
in the ID, for instance if no PMTs were selected for use in the ID but the track passes through a region easily visible to them, or if the track only crosses a very small amount of the IV. These penalties are used primarily to guide MINUIT into a reasonable parameter region and ideally are all small or zero for the final result.

\section{Fit Strategy}

\subsection{OV-ignored fit}

The tracks are parameterized by their position as they enter and exit the IV and their time when they enter the IV. The positions are represented by their polar and azimuthal angles, $\theta$ and $\phi$, with respect to the center of the detector. For the scintillator-dominated fit, the transition length parameter from Section 3.2 and the speed of light are also free parameters.

MINUIT is ultimately used to minimize the fit function, but cannot be relied upon to find the correct minimum from an arbitrary set of initial parameter values. Instead, to get a rough idea of where a track should be, the code first tests a table of uniformly distributed track guesses. For each guess, MINUIT is used to minimize the $\chi^{2}$ allowing only the muon entry time to vary. 194 guesses are used for scintillation-dominated events and 452 for Cherenkov-dominated events, where these numbers result from making uniform steps in $\theta$ and $\phi$ and selecting the results that intersect the correct detector volumes. If the density of these guesses is too low, the final results can erroneously cluster near the guesses themselves. With the density chosen, this effect is only significant when the number of selected PMTs is below 10. Although all of the guesses are downwards-going, MINUIT will, in the next step, not use this as a constraint. A small number of tracks are therefore reconstructed as upwards-going. The user can alternatively ask for an equal number of upwards-going guesses to be used. However, the rate of upwards-going muons in Double Chooz is negligible so this is not done by default.

The result with the lowest $\chi^{2}$ is used as input into a full fit. Even though at this point we expect to be near a local minimum in the fit function, and some attempt has been made to make the function smooth, it is nevertheless not smooth everywhere. We have found that MINUIT often fails to find the minimum via a single call to MIGRAD. Therefore the following progression of MIGRAD calls are used. First, all entry and exit angles are limited to $[-4 \pi, 4 \pi]$, which prevents pathologies when, for instance, $\theta$ is near zero while still giving MIGRAD quite a bit of freedom to choose its path towards the minimum. In contrast, if we were to limit $\theta$ to $[0, \pi]$ and $\phi$ to $[-\pi, \pi]$, it would be difficult to find minima near one of the limits. If MIGRAD does not immediately report success, it is called with successively higher values of its "tolerance" parameter until it does. This sequence helps negotiate kinks in the $\chi^{2}$ function due to physical boundaries such as moving the track from the lid of the GC to the side wall. Assuming this is achieved, the angle limits are lifted and MIGRAD is called once more as recommended by Ref. [9]. If the best $\chi^{2}$ happens to be a in a well-behaved region, errors are extracted using HESSE.

Finally, if the $\chi^{2}$ is more than three times the number of degrees of freedom or the $\mathrm{d} E / \mathrm{d} x$ in the ID of the resulting track is significantly lower than minimum ionizing, the entire procedure is repeated with a higher density of initial guesses in an attempt to find a better solution.

\subsubsection{Cherenkov Removal}

When doing a fit under the scintillation-dominated hypothesis, there may nevertheless be PMTs that cross the threshold before scintillation light hits them due to being near the muon entry or exit point and seeing a large amount of Cherenkov light. Near the exit point this is a mild effect, as the scintillation light and Cherenkov light arrive only a few nanoseconds apart, and the treatment of case (3) in Section 3.2 mostly takes care of the problem. However, PMTs near the entry point can see Cherenkov light much earlier than scintillation light since the Cherenkov light comes directly from the muon as soon as it enters the Buffer.

After doing the full fit procedure, the code checks for such PMTs. If found, they are removed and the fit is repeated. This is done up to two times, with up to three PMTs being removed from the fit each time. A PMT's timing is deemed to be due to Cherenkov light if it is at least $10 \mathrm{~ns}$ early compared to the fit expectation, or alternatively if it is at least $7 \mathrm{~ns}$ early, within $2 \mathrm{~m}$ of the track, and closer to the entrance in the Buffer than the exit.

This algorithm also eliminates other classes of early hits such as PMT pre-pulses and accidental coincidences with non-muon processes.

In the case of an intermediate amount of light (see Section 3.1) in which the choice between scintillation-dominated and Cherenkov-dominated is based on the fit results, the Cherenkov removal is only performed after this decision has been made. When there is OV data, Cherenkov removal is only done during the OV-ignored fit and the removed PMTs remain removed during the OV-inclusive fit. This is for performance reasons only and not a necessary feature.

\subsection{OV-inclusive fit}

If both the upper and lower panels of the OV provide $(x, y)$ coordinates, $O V$ tracks are formed. Typically more than one OV track is formed from a single muon due to crosstalk, bremsstrahlung, showering, etc. Since these tracks are very high resolution, we take it as given that one of them is correct and our only task is to choose between them. OV tracks can be rejected, however, if they miss the rest of the detector, or are severely inconsistent with the ID or IV signals. Each acceptable OV track is tested in turn by doing a fit that varies only the non-spatial track parameters (entry time, speed of light and transition length, as 
applicable). Using a combination of the resulting $\chi^{2}$ and the quality of the OV track, it selects one.

Usually, no OV tracks are present (or acceptable), but one of the two OV panels provides several possible sets of $(x, y)$ coordinates. The 6 of these with the largest energy deposition are considered for inclusion in the fit. Each is tested by repeating the fit with the track constrained to pass through the scintillator strips involved.

In some cases, the muon is only registered in one OV module and so the OV position is known very poorly. In this case, the fit is done with the loose constraint that the track must pass somewhere through the OV. While most low-quality OV triggers of this sort result from radioactivity rather than from muons, the fraction of these fits resulting from accidental coincidences is only $0.2 \%$.

As with the OV-ignored fit, a variety of initial conditions are tried, including both the result of the OV-ignored fit, adjusted as appropriate, and a fixed table of uniformly distributed guesses. For use by MINUIT, the spatial component of the track is parameterized by the $x$ and $y$ position within the OV and the $\theta$ and $\phi$ of the track. In the $\chi^{2}$ function, this is translated into the representation described in section 4.1 so that no code is duplicated.

As above, the code is usually configured to assume that all muons are downwards-going, but can also attempt upwards-going if requested. In this case, the entire procedure is done each way and the overall result with the lower $\chi^{2}$ is returned.

\subsection{Stopping Muons}

If the muon deposited an unusually low amount of energy in the IV, it is a stopping muon candidate. All muonlike events with less than $70 \%$ of the mean muon IV energy are reconstructed under both through-going and stopping hypotheses. Because it is not possible to know the expected amount of light produced in the IV without first knowing the muon trajectory, this is a loose cut that certainly covers all muons that stop in the ID while still excluding most through-going muons to save processing time.

The stopping muon fit function that MINUIT minimizes is the same as the through-going scintillation-dominated fit, except that:

- Light is only expected from the entry point into the IV rather than at two points.

- An additional free parameter is added to the fit: the fraction of the GC and NT crossed along the track trajectory. No light is produced past this point.

- The method described in Section 3.2 to smooth out the scintillator entry points is applied to both the entry point and the stopping point, since there is no Cherenkov light from the Buffer after the exit point if the muon does not exit. The muon track becomes visible to the PMTs below the stopping point much more rapidly than to those above the entry point due to the scintillation light piling up behind the muon. Because of this, a shorter transition length is used at the stopping point.

- The track length in the ID is strongly constrained to match the observed ID energy using a $\chi^{2}$ penalty term. Unlike through-going muons, stopping muons do not have enough energy to shower and should have a one-to-one correspondence between track length and visible energy.

The muon is still assumed to travel at the speed of light up to the stopping point. This is a good approximation: only in the last $250 \mathrm{~mm}$ does it drop below $250 \mathrm{~mm} / \mathrm{ns}$.

The overall fitting strategy is the same as for the through-going scintillation-dominated fit, except that the speed of light and the transition length parameter are not allowed to float since allowing them to float was found to worsen the resolution. The same density of initial track guesses is used. For the initial fits in which only the muon entry time is allowed to vary, the stopping position parameter is fixed at the value that produces the right scintillator path length for the observed energy. This fit is done both with and without the OV if OV data is present.

\subsection{Performance}

On a $2.4 \mathrm{GHz}$ Intel Xeon CPU, the mean time used per muon is $0.12 \mathrm{~s}$. At the Double Chooz far detector rate, this means about $6 \mathrm{CPU}$-hours are needed to reconstruct 1 hour of data. About half of the runtime is used doing one-parameter time-only fits to determine the correct starting point for each type of fit, as described in Section 4.1. The other half is used doing the full fits from the favored starting points.

\section{Choice of Fit}

As described, up to four fits are done - all combinations of OV-exclusive/inclusive and through-going/stopping. One is selected as the best answer. For the throughgoing fits, if there is OV data, the OV-inclusive fit is usually judged to be better than the OV-ignored fit on the basis that the OV position information is relatively unambiguous. However, since it is possible for the OV signal to be due to an accidental coincidence, or for the OV hits to be from secondaries rather than the muon itself, from other muons originating in the same air shower, or for there to be a pathology in the $\mathrm{OV}$-inclusive reconstruction, the $\mathrm{OV}$-ignored fit is selected if its $\chi^{2}$ is at least 1000 units lower.

If a stopping muon fit was done, it is chosen as the best answer if the IV energy is consistent with a single IV crossing, given the reconstructed length of IV scintillator traversed (see Fig. 5). If OV information is present and the stopping fit is chosen, the OV-inclusive fit is always chosen. There is no option to reject this fit in favor of the OV-ignored fit because the low statistics of the stopping 


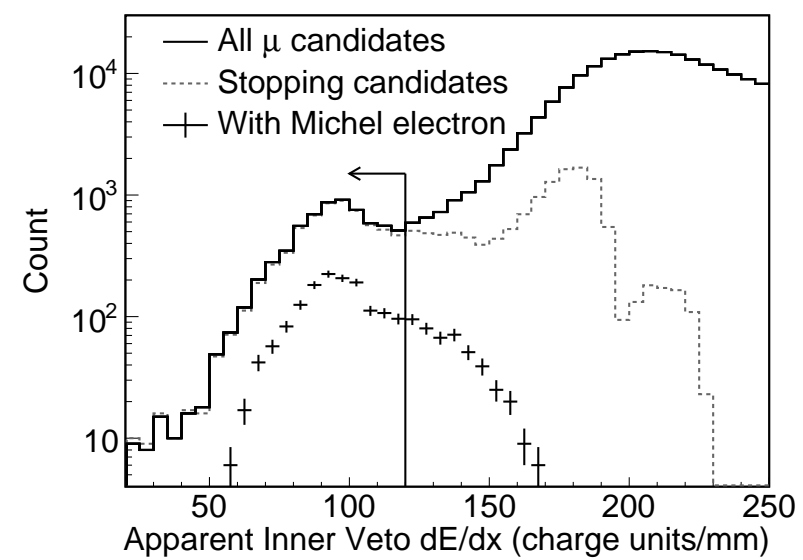

Figure 5: $\mathrm{d} E / \mathrm{d} x$ in the IV for muons fit under the through-going hypothesis. The solid line shows all muons. Through-going minimum ionizing muons make up the broad peak around 210. The dashed line shows muons fit under the stopping muon hypothesis. The stopping muon hypothesis is favored if $\mathrm{d} E / \mathrm{d} x<120$. Finally, muons followed by Michel electrons are shown. The efficiency for observing these is low. In particular, electrons produced in the Buffer are lost.

muon sample make it difficult to determine when, if ever, this would be beneficial.

While one of the fits is chosen as the best, all four fit results are also saved separately. This is useful since some analyzers may wish, for instance, to use the OV-ignored fit more often to exclude accidental coincidences, or, in the case of through-going versus stopping, one may wish to apply a different cut to obtain either a larger sample or a more pure one. It also allows the reconstruction to be tuned by comparing the agreement of the OV-ignored fits to the OV data.

\section{Self-calibration}

Double Chooz has two light injection systems used for timing calibration, a multiwavelength LED-fiber system installed on the buffer walls [1], and a $470 \mathrm{~nm}$ laser diffuser which is deployed along the vertical axis. The latter is more precise, producing time constants with an uncertainty of $0.15 \mathrm{~ns}$; the two systems are found to give consistent results within $0.5 \mathrm{~ns}$. We find that with the calibrations derived from these systems applied, the per-PMT muon fit residuals are typically offset by up to $1 \mathrm{~ns}$. The muon statistics available in each hour-long run are sufficient to measure these offsets with a precision of $0.02 \mathrm{~ns}$. These offsets are believed to be a consequence of the reconstruction's imperfect model of muon light distribution coupled with the fact that different PMTs tend to preferentially sample different parts of the distribution.

To correct for this, the residuals are used to produce a new set of timing calibrations using an iterative procedure. Because the Double Chooz far detector is under a hill, the muon flux is not uniform as a function of the azimuthal angle. Calibration events are weighted so that all azimuthal

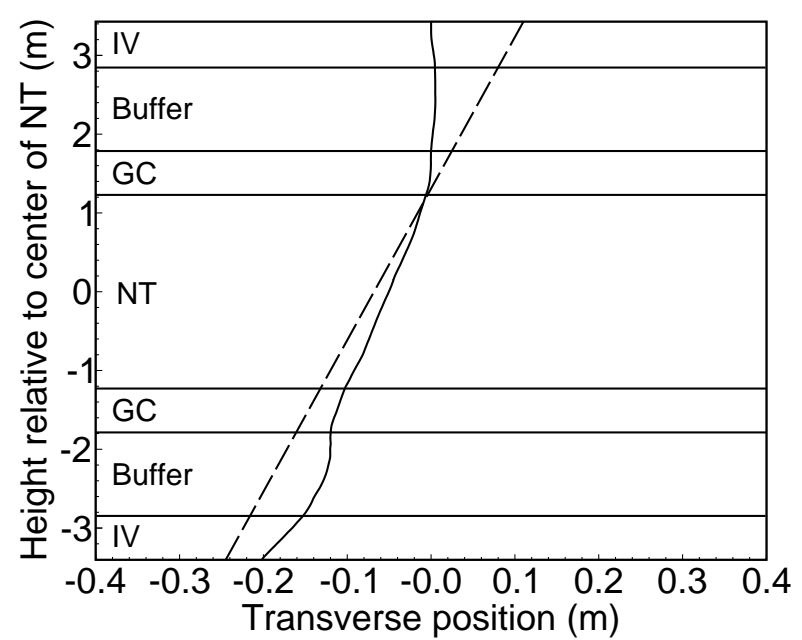

Figure 6: Reconstruction of a low energy muon. The solid line is a simulated muon track. The muon initially has a kinetic energy of $1.5 \mathrm{GeV}$ and is going straight down. It scatters through $0.2 \mathrm{~m}$ as it traverses the detector (note exaggerated horizontal scale). The dashed line shows the reconstructed track.

angles are equally represented. If we do not perform this weighting, the fit resolution suffers and the fit tracks are biased towards the direction of the average muon.

The self-calibration is done for each 1-hour run. It improves the resolution by $16 \%$ while also providing the best measure of PMT timing stability for Double Chooz, since no dedicated calibration runs are needed.

\section{Resolution}

The resolution of the fit is tested using data-driven methods, primarily in events with OV tracks. The resolution of the OV-ignored fit can be tested by comparing these fits with OV tracks. In the same way, the resolution of fits done with $(x, y)$ coordinates in a single OV panel can be tested. While the acceptance of OV tracks is small due to the limited size of the upper OV, the symmetry of the ID suggests that resolutions measured using this method are generally valid. One can also compare OV-ignored fit fits to hits in the lower OV panel alone when OV tracks are not available. This provides limited information, but a much larger acceptance. Results from this method agree with that of OV track comparison.

If the resolution were limited by PMT timing alone, we would expect, in the ideal case of a minimum ionizing through-going muon intersecting a large amount of ID scintillator ( $8 \%$ of the reconstructed muons), to obtain a resolution of about $20 \mathrm{~mm}$ in each transverse coordinate at the detector center. In other words, this is the error that MINUIT reports. At low muon energies, the error must increase since the fit assumes that muons travel in straight lines, while real muons undergo multiple scattering (see Fig. 6). The median muon energy at the Double Chooz 


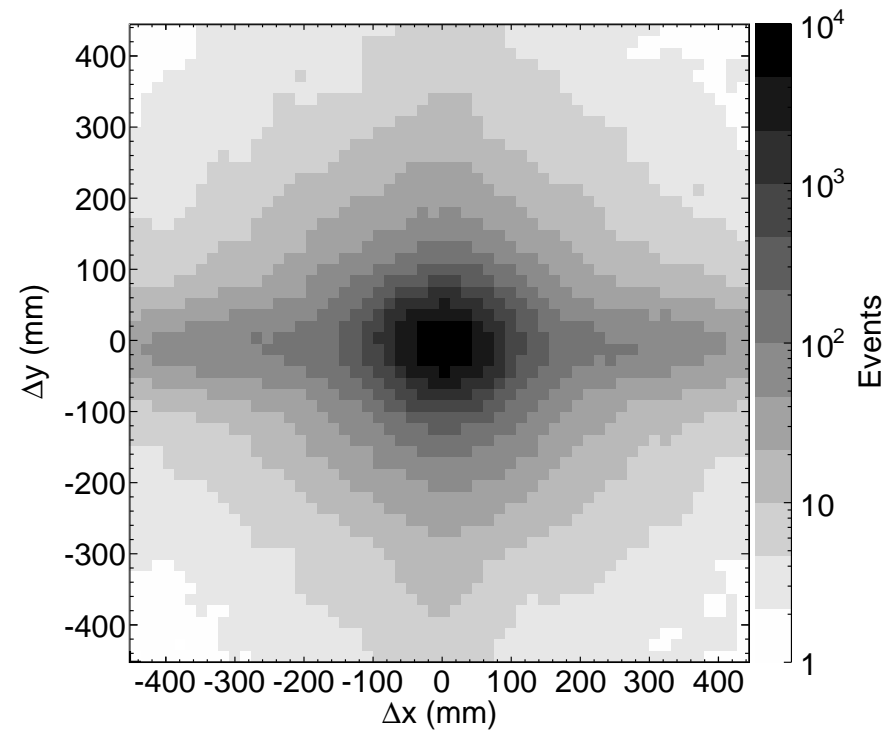

Figure 7: Resolution as measured by OV tracks. Shown is the relative position in the $z=0$ plane between the OV track and the result of this reconstruction without use of OV data. Only events in which all ID PMTs are used are plotted here. This OV's own resolution is a significant effect. The ridge along $\Delta y=0$ arises from OV track misreconstructions and the asymmetry of the OV.

far detector is about $30 \mathrm{GeV}$; at this energy the RMS deviation due to multiple scattering as a muon crosses the ID is $40 \mathrm{~mm}$ in each transverse coordinate. By comparison with OV tracks, we find that the resolution is about $40 \mathrm{~mm}$ at the center of the detector in this case (see Fig. 7). The resolution is larger at the top and bottom of the detector by about a factor of 2, as shown in Fig. 8. This figure compares the resolution if the OV is not used to that obtained with either the upper or lower OV panel alone, and to the resolution of OV track. OV hits are present in about half of events across all categories of muons, and OV tracks in $6 \%$ of events. All resolutions are shown after subtracting off the resolution of OV tracks, which is estimated from the OV strip widths and the effects of multiple scattering.

The resolution gradually worsens as the muon's path length through the detector decreases. The addition of OV information becomes more important as fewer PMTs are used. The lowest energy scintillator-dominated events are fit with a resolution of about $100(150) \mathrm{mm}$ with(without) OV hits. Muons that pass only through the IV and Buffer - Cherenkov events, $35 \%$ of the reconstructed muons are fit with an $x$ and $y$ resolution of typically $200 \mathrm{~mm}$ at the center of their path without OV hits, or $150 \mathrm{~mm}$ when the OV is used. The $29 \%$ of muons passing only through the IV are reconstructed with a resolution of $250 \mathrm{~mm}$ at the corner of the IV that they intersect, but very little angular information can be obtained without the OV and so the resolution is very poor at any other height. With OV hits, the $x$ and $y$ resolution are better than $1 \mathrm{~m}$ everywhere above the bottom of the detector.

The presence of a shower distorts the fit by adding ad-

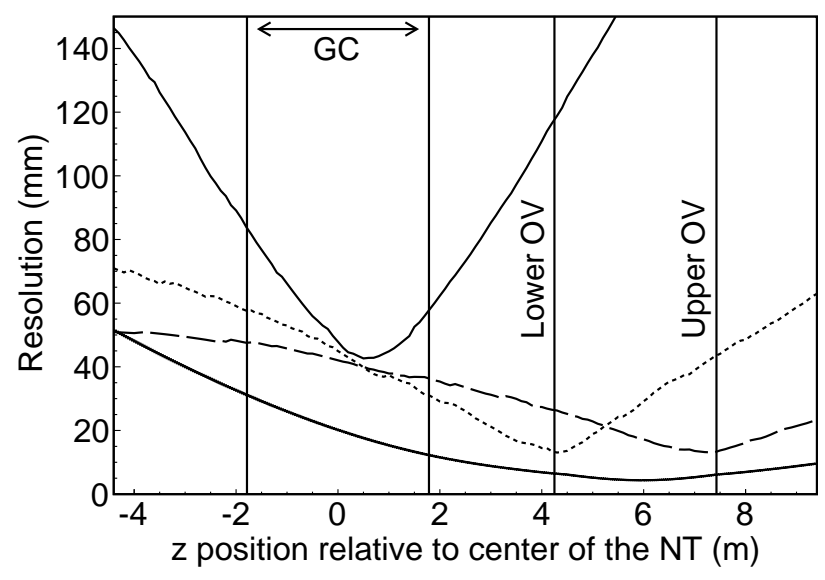

Figure 8: Resolution along reconstructed tracks as a function of vertical position for events with OV tracks and all ID PMTs used. The upper solid line gives the resolution if the OV is ignored. The dashed (dotted) line gives the resolution if only the upper (lower) OV is used. The lower solid line is the resolution of the OV track. The vertical extent of the GC and positions of the OV panels are shown.

ditional light away from the muon track. However, if the shower begins inside the detector, this light can only lead the muon's own light by a small amount, and can sometimes be rejected by the Cherenkov removal procedure. Resolution for showering muons is typically $10-40 \%$ worse than for minimum ionizing muons, depending on the extent of the shower. The most important application of muon tracking in Double Chooz, ${ }^{9} \mathrm{Li} /{ }^{8} \mathrm{He}$ identification, is affected by this loss of resolution since these isotopes are produced by showering muons. However, the typical physical distance between the muon and the ${ }^{9} \mathrm{Li} /{ }^{8} \mathrm{He}$ production is still greater than the resolution, so the ability to identify them remains excellent.

The accuracy of the stopping muon fit was tested by comparing the reconstructed stopping position to the location of the following Michel electron. The resolution was found to be $150 \mathrm{~mm}$ in each of $x, y$ and $z$. Interestingly, while stopping muon fits done using $(x, y)$ coordinates from a single OV panel to constrain the track have somewhat better resolution than those without OV data, fits done with an OV track have significantly worse resolution. This is because the muon's path typically deviates significantly from a straight line as it comes to a stop. While the fit always assumes a straight-line path, if allowed to move it finds a better approximation to the true path than that provided by the OV track.

\section{Conclusions}

We have developed a sophisticated muon reconstruction algorithm for Double Chooz that provides resolution sufficient for several physics goals. Double Chooz's OV was instrumental in the development of this reconstruction and provides substantial improvement to its resolution for 
tracks crossing the OV layers. The techniques presented here are applicable, in whole or in part, to any similar detector, such as those operated by the currently-running Daya Bay [10] and RENO [11] experiments, or planned detectors such as LENA [12], JUNO [13], RENO-50 [14], and $\mathrm{SNO}+[15]$.

\section{Acknowledgments}

We thank the French electricity company EDF; the European fund FEDER; the Région de Champagne Ardenne; the Département des Ardennes; and the Communauté des Communes Ardennes Rives acknowledge the support of the CEA, CNRS/IN2P3, the computer center CCIN2P3, and LabEx UnivEarthS in France (ANR-11-IDEX-000502); the Ministry of Education, Culture, Sports, Science and Technology of Japan (MEXT) and the Japan Society for the Promotion of Science (JSPS); the Department of Energy and the National Science Foundation of the United States; the Ministerio de Ciencia e Innovación (MICINN) of Spain; the Max Planck Gesellschaft, and the Deutsche Forschungsgemeinschaft DFG (SBH WI 2152), the Transregional Collaborative Research Center TR27, the excellence cluster "Origin and Structure of the Universe", and the Maier-Leibnitz-Laboratorium Garching in Germany; the Russian Academy of Science, the Kurchatov Institute and RFBR (the Russian Foundation for Basic Research); the Brazilian Ministry of Science, Technology and Innovation (MCTI), the Financiadora de Estudos e Projetos (FINEP), the Conselho Nacional de Desenvolvimento Científico e Tecnológico (CNPq), the São Paulo Research Foundation (FAPESP), and the Brazilian Network for High Energy Physics (RENAFAE) in Brazil.

[1] Y. Abe, et al. (Double Chooz Collaboration), Reactor electron antineutrino disappearance in the Double Chooz experiment, Phys.Rev. D86 (2012) 052008.

[2] T. Matsubara, T. Haruna, T. Konno, Y. Endo, M. Bongrand, et al., Evaluation of 400 low background 10-in. photo-multiplier tubes for the Double Chooz experiment, Nucl.Instrum.Meth. A661 (2012) 16-25.

[3] C. Bauer, E. Borger, R. Hofacker, K. Janner, F. Kaether, et al., Qualification Tests of 474 Photomultiplier Tubes for the Inner Detector of the Double Chooz Experiment, JINST 6 (2011) P06008.

[4] E. Calvo, M. Cerrada, C. Fernandez-Bedoya, I. Gil-Botella, C. Palomares, et al., Characterization of large area photomutipliers under low magnetic fields: Design and performances of the magnetic shielding for the Double Chooz neutrino experiment, Nucl.Instrum.Meth. A621 (2010) 222-230.

[5] D. Dietrich, D. Greiner, J. Jochum, T. Lachenmaier, M. Rohling, et al., Monte Carlo aided design of the inner muon veto detectors for the Double Chooz experiment, JINST 7 (2012) P08012.

[6] L. A. Winslow, First solar neutrinos from KamLAND: A measurement of the beryllium-8 solar neutrino flux, Ph.D. thesis, University of California, Berkeley, 2008.

[7] Y. Abe, et al. (Double Chooz Collaboration), First Measurement of $\theta_{13}$ from Delayed Neutron Capture on Hydrogen in the Double Chooz Experiment, Phys.Lett. B723 (2013) 66-70.

[8] Y. Abe, T. Akiri, A. Cabrera, B. Courty, J. Dawson, et al., The Waveform Digitiser of the Double Chooz Experiment: Performance and Quantisation Effects on PhotoMultiplier Tube Signals, JINST 8 (2013) P08015.
[9] F. James, M. Roos, Minuit: A System for Function Minimization and Analysis of the Parameter Errors and Correlations, Comput.Phys.Commun. 10 (1975) 343-367.

[10] F. An, et al. (Daya Bay Collaboration), Improved Measurement of Electron Antineutrino Disappearance at Daya Bay, Chin.Phys. C37 (2013) 011001.

[11] J. Ahn, et al. (RENO collaboration), Observation of Reactor Electron Antineutrino Disappearance in the RENO Experiment, Phys.Rev.Lett. 108 (2012) 191802.

[12] M. Wurm, et al. (LENA Collaboration), The next-generation liquid-scintillator neutrino observatory LENA, Astropart.Phys. 35 (2012) 685-732.

[13] Y.-F. Li, J. Cao, Y. Wang, L. Zhan, Unambiguous Determination of the Neutrino Mass Hierarchy Using Reactor Neutrinos, Phys.Rev. D88 (2013) 013008.

[14] S.-H. Seo, Observation of Reactor Antineutrino Disappearance at RENO and Future Plan, Nucl.Phys.Proc.Suppl. 237-238 (2013) 65-70.

[15] J. Maneira (SNO+ Collaboration), The SNO+ experiment: status and overview, J.Phys.Conf.Ser. 447 (2013) 012065. 